\title{
The Most Trustworthy Path Selection in Online Social Networks
}

\author{
Lianggui Liu \\ School of Information Science and Technology, Zhejiang Sci-Tech University, Hangzhou 310018, China \\ Correspondence should be addressed to Lianggui Liu; lgliu@hotmail.com
}

Received 22 June 2013; Accepted 19 September 2013

Academic Editor: Miguel A. F. Sanjuán

Copyright (c) 2013 Lianggui Liu. This is an open access article distributed under the Creative Commons Attribution License, which permits unrestricted use, distribution, and reproduction in any medium, provided the original work is properly cited.

\begin{abstract}
In online social networks, it is crucial for a service consumer to find the most trustworthy path to a target service provider from numerous social trust paths between them. The selection of the most trustworthy path (namely, optimal social trust path (OSTP)) with multiple end-to-end quality of trust (QoT) constraints has been proved to be NP-Complete. Heuristic algorithms with polynomial and pseudo-polynomial-time complexities are often used to deal with this challenging problem. However, existing solutions cannot guarantee the efficiency of searching; that is, they can hardly avoid obtaining partial optimal solutions during searching process. Quantum annealing uses delocalization and tunneling to avoid falling into local minima without sacrificing execution time. It has been proved to be a promising way to many optimization problems in recently published literature. In this paper, for the first time, QA based OSTP algorithm (QA_OSTP) is applied to the selection of the most trustworthy path. The experiment results show that QA based algorithm has better performance than its heuristic opponents.
\end{abstract}

\section{Introduction}

Online social networks (OSNs) [1,2] have gained much attention from the world recently. According to Nielson's report in 2009 , more than two-thirds of the global online population visit and participate in online social networks [3]. In such kind of networks, the network structure is made up of participants such as individuals or organizations and links between participants such as interactions, relationships, and connections. Since there may be over thousands of paths between a pair of interactive participants in large scale OSNs [4], a primary concern arise spontaneously, that is, for a source participant (e.g., a service consumer), how to exactly find the most trustworthy path to a target participant (e.g., a service provider) within a tolerable period of time? Unfortunately, the selection of the most trustworthy path, in other words, optimal social trust path (OSTP) selection with multiple end-toend quality of trust (QoT) constraints, has been proved to be NP-Complete [5]. Evaluating the trustworthiness of the target participant along all these social trust paths requires large amount of computation time [6]. Polynomial and pseudopolynomial-time heuristic algorithms are often used to deal with this problem. However, existing solutions cannot guarantee the quality of final configuration or the speed of searching. Thus, this field is attracting more and more attention recently with the rapid development of OSNs.

\section{Related Work}

2.1. Multiple QoS Constrained Path Selection. Korkmaz and Krunz [7] firstly proposed an approximation algorithm H_MCOP to determine a feasible path that satisfies a set of constraints while maintaining high utilization of network resources. In their algorithm, both multiconstrained values and values of QoS parameters values are aggregated based on

$$
g_{\lambda}(p) \stackrel{\operatorname{def}}{=}\left(\frac{w_{1}(p)}{c_{1}}\right)^{\lambda}+\left(\frac{w_{2}(p)}{c_{2}}\right)^{\lambda}+\cdots+\left(\frac{w_{K}(p)}{c_{K}}\right)^{\lambda}
$$

where $\lambda \geq 1 ; w_{i}(p)$ is the aggregated value of the $i$ th QoS parameter of path $p ; c_{i}$ is the $i$ th QoS constraint value of the selected path between source node $s$ and destination node $d$. H_MCOP firstly uses Dijkstra's algorithm to find the path with the minimum $g_{\lambda}$ from $s$ and $d$, which intends to find whether there exists a feasible solution satisfying all end-toend QoS parameters in a subnetwork. During this process, the aggregated value of each QoS parameter for the identified path from $s$ to $d$ is computed and recorded at each intermediated node along the path. If there exists at least one feasible solution, then these aggregated values are used in another search from $s$ to $d$, which intends to identify a feasible path from $s$ to $d$ with the minimal cost. 
Yu et al. [8] studied the problem of service selection with multiple QoS constraints and proposed an approximation algorithm, MCSP-K based on H_MCOP, which checks the number of paths it currently keeps and removes the path with the maximum $g_{\lambda}$ if the path number reaches $K$. In their algorithm, the paths with $K$ minimum $g_{\lambda}$ will be kept at each intermediate node, which will ensure that MCSP-K will never prune out a feasible path if one exists. In the service candidate graph therein, there is a link between any two nodes in adjacent service sets. If these requirements cannot be satisfied in a network, MCSP_K will search all the paths from source node to each intermediate that will lead the time complexity to be exponential. Thus, this algorithm does not fit for large scale online social networks.

2.2. Heuristic Algorithm for OSTP. Until now, there are only a few works that are proposed to address the problem in online social networks where some significant influence factors including trust, recommendation roles, and social relationships are taken into account.

Liu et al. [5] developed a novel efficient heuristic algorithm for OSTP selection named MFPB_HOSTP in online social networks based on Dijkstra's algorithm [9]. In MFPB_ HOSTP, they first proposed the objective function given in

$$
\delta(p) \stackrel{\text { def }}{=} \max \left\{\left(\frac{1-T_{p}}{1-c_{1}}\right),\left(\frac{1-r_{p}}{1-c_{2}}\right),\left(\frac{1-\rho_{p}}{1-c_{3}}\right)\right\},
$$

where $T_{p}, r_{p}, \rho_{p}$ are QoT parameters and represent trust information between participants, social relationships between adjacent participants, and recommendation roles of a participant, respectively. They adopted the Backward_Search procedure to identify the path with the minimal $\delta$ from $s$ to $d$ to investigate whether there exists a feasible solution where all end-to-end QoT constraints can be satisfied in the subnetwork and to record the aggregated QoT parameters of the path identified from $s$ to each intermediate node. If a feasible solution exists, MFPB_HOSTP then adopts the Forward_Search procedure to find a near-optimal path from $s$ to $d$. This process adopts the information provided by Backward_Search to identify whether there is another path that satisfies QoT constraints. In this process, MFPB_HOSTP first searches the path with maximal $\mathscr{F}$ value from $s$. Assume one of the adjacent nodes of $s, m$ is selected based on Dijkstra's shortest path algorithm as the utility of the path from $s$ to it is maximal. Let $p_{m \rightarrow d}^{b(\delta)}$ denote the backward local path from $m$ to $d$ identified in the Backward_Search procedure. Then a foreseen path from $s$ to $d$ via $m$ is formed. If this foreseen path is feasible, then MFPB_HOSTP chooses the next node from $m$ with the maximal $\mathscr{F}$ value that is calculated based on Dijkstra's shortest path algorithm. Otherwise, MFPB_HOSTP does not search the path from $m$ and the link $s \rightarrow m$ will be deleted from the subnetwork. Subsequently, MFPB_HOSTP performs the Forward_Search procedure to search the path from $s$ in the subnetwork without the link $s \rightarrow m$. MFPB HOSTP is one of the most promising algorithms in solving the OSTP selection problem as it outperforms prior exiting algorithms in both efficiency and the quality of delivered solutions [5].
2.3. Quantum Annealing (QA). In statistical mechanics, a physical process called simulated annealing (SA) is often performed in order to relax the system to the state with the minimum energy. In the basic form of SA, it first generates an initial solution as the current feasible solution using Metropolis algorithm $[10,11]$. Then another solution is selected in the neighborhood of the current solution and replaces the current solution with the new one with the following transition probability given by Metropolis criterion:

$$
P(i \Longrightarrow j)= \begin{cases}1 & \text { if } f(j) \leq f(i) \\ \exp \left(\frac{f(i)-f(j)}{t}\right) & \text { otherwise }\end{cases}
$$

where $t \in R^{+}$represents the control parameter. $f(i)$ and $f(j)$ are energy functions corresponding to state $i$ and $j$, respectively. The same process continues iteratively many times. Nonoptimal configuration with probability $\exp ((f(i)-f(j)) /$ $t$ ) is used to avoid being stuck in a local optimization each time, although the goal is to find a global optimal configuration. Obviously, result of one arbitrary taste is only dependent upon the result of the previous taste; thus, concepts in a Markov Chain corresponding to a control temperature $t$ can be used. As to SA, one-step transition matrix in a Markov Chain is defined as follows:

$$
\begin{aligned}
& P_{t}(i, j) \\
& =\operatorname{Pr}[s(q+1)=j \mid s(q)=i] \\
& = \begin{cases}0 & \text { if } j \notin N(i), j \neq i \\
G(i, j) \min \left\{1, e^{(f(i)-f(j)) / T}\right\} & \text { if } j \in N(i), j \neq i \\
1-\sum_{i^{\prime} \neq i} G\left(i, i^{\prime}\right) \min \left\{1, e^{(f(i)-f(j)) / T}\right\} & \text { otherwise, }\end{cases}
\end{aligned}
$$

where $G(i, j)$ represent the probability with which configuration $j$ is derived from $i$ and $N(i) \subseteq S$ is the neighborhood set of $i$. With the temperature decreasing, only the better deterioration configuration can be accepted. Simulated annealing is a particularly promising minimization technique. For example, it has proved effective in finding the global minimum of multidimensional functions having large numbers of local minima [12].

It is worth mentioning that introducing thermal fluctuation is not the only way to perform annealing; QA depends on quantum fluctuation instead [13]. A prominent advantage of quantum fluctuation over thermal fluctuation originates from the fundamental property of the quantum theory, namely, the possibility of tunneling through classically impenetrable potential barriers between energy valleys. Consequently, methods of quantum search, in principle, could be more efficient than the classical search methods [14-17]. Furthermore, the effect of quantum tunneling is shown to be crucial for solving many computationally difficult problems, including the class of nondeterministic polynomial time problems. A practical implementation of QA will need to solve the timedependent Schrödinger equation in a very large and exponentially growing Hilbert space, which can only rely on a robust 
quantum computer. Recent research in this area has been carried out by Green's Function Monte Carlo (GFMC) simulations using quantum-classical mapping with the aid of a Suzuki-Trotter transformation [18, 19], inspired from which we apply QA to the OSTP in OSNs. To the best of our knowledge, this is the first application of QA to the OSTP, and experiment results show that QA based searching algorithm has better performance than its heuristic opponent.

The structure of the present paper is as follows. In Section 3, we describe our proposed algorithm in detail. Specifically, in Section 3.1 Quantum Hamiltonian is given, which is then approximated by a classical one with the aid of a SuzukiTrotter transformation. The QA based OSTP algorithm (QA_ OSTP) is presented and efficiency concerns are addressed in Sections 3.2 and 3.3, respectively. Section 4 contains experimental results and analysis, which show that QA_OSTP outperforms MFPB_HOSTP. Finally, we conclude this paper in Section 5 with a summary and research prospects.

\section{Algorithm Description}

In order to give prominence to the main problem of OSTP in online social networks, the Enron email dataset [20], a widely used system in the investigation of social networks [5, 21-23], is selected for our research, and the related parameter sets such as QoT set and set of weight of QoT parameters are the same as those in [5] for comparison.

In order to solve OSTP in large scale online social networks using quantum annealing, we first need to map the problem onto a highly constrained Ising model [24, 25]. Then searching the optimal social trust path is corresponding to finding the ground state with the lowest energy in an Ising model. Moreover, some assumptions should be given first as follows to eliminate some secondary factors that will increase unnecessary complexity and may influence the performance of the online social networks.

Assumption 1. Before searching the OSTP, three QoT parameters, that is, the values of trust, the social intimacy degree between participants, and the role impact factor of participants mentioned in [5], have been already obtained through mining techniques [22].

Assumption 2. Since the transverse Ising spin glass (TISG) model is the simplest model in which quantum effects in a random system can and have been studied extensively and systematically [26], here we focus only on the TISG.

Assumption 3. The OSNs studied here are symmetric. Symmetric means that each pair of adjacent participants, truster and trustee, can reverse their roles without changing their trust values. For example, in Figure 1, we set $T_{\mathrm{AC}}=T_{\mathrm{CA}}$, $r_{\mathrm{AC}}=r_{\mathrm{CA}}$.

3.1. Problem Representation. In a TISG model, phase space is spanned by all the set of spin variables $\left\{\omega_{\chi}\right\}$, each of which corresponds to a possible configuration.

(1) Quantum Hamiltonian. In QA, the total Hamiltonian for the OSTP can be expressed as a time-dependent Hamiltonian:

$$
H_{\mathrm{OSTP}}=H_{\mathrm{pot}}+H_{\mathrm{kin}}
$$

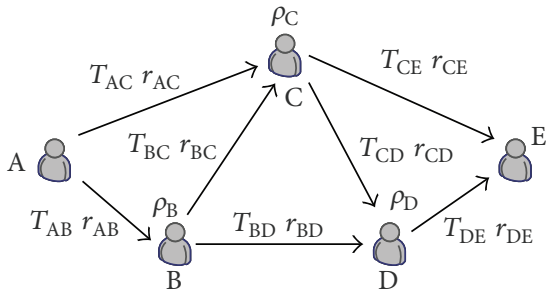

FIGURE 1: Online social networks.

where $H_{\text {pot }}$ represents the classical potential energy of a given configuration and $H_{\text {kin }}$ is a suitable kinetic energy operator providing the necessary quantum fluctuations to escape local minima. In QA, we seek to minimize $H_{\text {pot }}$ as side effect of minimizing $H_{\text {OSTP }}$. A suitable configuration is reached if and only if $H_{\text {pot }}$ is zero.

In TISG model, the total Hamiltonian in (5) can be rewritten as

$$
H_{\mathrm{OSTP}}=H_{\mathrm{TISG}}+H_{\mathrm{TF}}(t),
$$

where $H_{\text {TISG }}$ denotes potential energy of TISG model and $H_{\mathrm{TF}}(t)$ is a fictitious kinetic energy introduced typically by the time-dependent transverse field.

A TISG model consists of a set of spins, each of which can only be in one of two states. Each of these spin variable usually takes on the value of either +1 or -1 , also known as an up-spin and a down-spin, respectively. Formally, for online social networks with $N$ participants each configuration of the system (a feasible social trust path) is associated to a $N \times N$ matrix $U$ with $0 / 1$ entries in the following way: for each pair of the participants $i$ and $j$, if the directed social trust path (an ordered sequence of participants in online social networks) goes through the link between $i$ and $j$, then $U_{i j}=1$, or else $U_{i j}=0$. Here we renumber the independent variables as $U_{k}(k=1, \ldots, K)$ and the other dependent variables can be expressed by $U_{k}$. Then, in quantum mechanics, the quantum Hamiltonian of the OSTP can be expressed as a $K$-spins TISG model through the transformation $U_{k} \rightarrow\left(1+\sigma_{z}^{k}\right) / 2$, where $\sigma_{z}^{k}$ is the Pauli matrix of qubit $k$. Moreover, the possible social trust paths can be represented by different quantum states of the $K$ spins

$$
H_{\mathrm{TISG}} \equiv \sum_{i=1}^{K} v_{i} \sigma_{z}^{i}+\sum_{i=1}^{K} \sum_{j=i+1}^{K} J_{i j} \sigma_{z}^{i} \sigma_{z}^{j},
$$

where $m$ and $n$ represent two randomly selected participants, that is, two qubits in TISG model, $v_{m}$ is the off-resonance term related to $m, \sigma_{z}^{m}$ and $\sigma_{z}^{n}$ describe the Pauli matrix of qubit $m$ and $n$, and $J_{m n}$ denotes the spin-spin coupling between qubit $m$ and $n$.

We use Figure 2 and Table 1 as a demonstration to describe this kind of correspondence.

In Figure 2, OSNs are composed by four participants, where all the parameters are the same as those defined in [5]. There are two possible social trust paths between participant 1 and participant 4 , that is, $1-3-4$ and $1-2-4$. The utility values of these two paths determined by the utility function in 
TABLE 1: Possible social trust path and corresponding state vector in Figure 2.

\begin{tabular}{lccc}
\hline$U_{i j}$ & Social trust path & State vector & Utility \\
\hline$U_{12}=1$ & $1-2-4$ & $|01\rangle$ & 0.744 \\
$U_{13}=0$ & & & \\
$U_{12}=0$ & $1-3-4$ & $|10\rangle$ & 0.384 \\
$U_{13}=1$ & & $|11\rangle$ & Invalid \\
$U_{12}=1$ & Invalid path & & \\
$U_{13}=1$ & & $|00\rangle$ & Invalid \\
$U_{12}=0$ & Invalid path & & \\
$U_{13}=0$ & & &
\end{tabular}

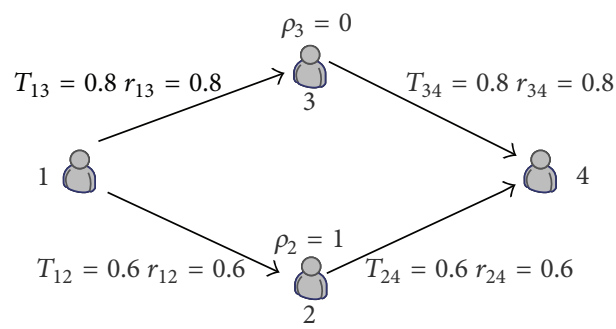

Figure 2: An example of OSNs $\left(w_{T}=0.3, w_{r}=0.3, w_{\rho}=0.4\right)$.

[5] are 0.384 and 0.744 , respectively. Thus even path $1-3-4$ has larger trust value and $r$, participant 1 will choose $1-2-4$ as its preferred social trust path according to the weights specified by itself.

Note that the realization of quantum annealing requires introducing an artificial and adjustable quantum kinetic operator that can provide the quantum fluctuations to escape the local minima. Moreover, the quantum annealing process is required to be slow enough to approximate the adiabatic evolution. Reasonably, the choice of $H_{\text {kin }}$ should encompass one important question that is determining which configurations are to become direct neighbors of a given configuration. Here we use an effective tactics named "plus-minus" [27] as disturbance mechanism to realize a neighborhood of a given configuration. Then in TISG model, to implement QA, a fictitious kinetic energy is introduced typically by the timedependent transverse field:

$$
H_{\text {Kin }} \equiv \Gamma(t) \sum_{i=1}^{K} S_{\left\langle i^{\prime}, i\right\rangle}^{+} S_{\left\langle j^{\prime}, j\right\rangle}^{+} S_{\langle j, i\rangle}^{-} S_{\left\langle j^{\prime}, i^{\prime}\right\rangle}^{-}
$$

where $\Gamma(t)$ is the time-dependent power of the transverse field, each $S_{\langle i, j\rangle}^{ \pm}$flips an Ising spin variable at position $(i, j)$ and at the symmetric position $(j, i)$, that is, $S_{\langle i, j\rangle}^{ \pm}=S_{i, j}^{ \pm} S_{j, i}^{ \pm}$. Then we have the following form of the total quantum Hamiltonian of OSTP:

$$
\begin{aligned}
H_{\mathrm{OSTP}}= & \sum_{i=1}^{K} v_{i} \sigma_{z}^{i}+\sum_{i=1}^{K} \sum_{j=i+1}^{K} J_{i j} \sigma_{i}^{z} \sigma_{z}^{i} \sigma_{z}^{j} \\
& +\Gamma(t) \sum_{i=1}^{K} S_{\left\langle i^{\prime}, i\right\rangle}^{+} S_{\left\langle j^{\prime}, j\right\rangle}^{+} S_{\langle j, i\rangle}^{-} S_{\left\langle j^{\prime}, i^{\prime}\right\rangle}^{-} .
\end{aligned}
$$

Initially the strength of the transverse field $\Gamma(t)$ is chosen to be very large, and $H_{\text {OSTP }}$ is dominated by the third term of (9). Then $\Gamma(t)$ is gradually and monotonically decreased toward zero, leaving eventually only the first two terms. Note that every state of TISG model can be described as state vector $|\psi(t)\rangle$, and it will evolve with time and should follow the RT Schrödinger equation,

$$
i \frac{d}{\mathrm{dt}}|\psi(t)\rangle=H(t)|\psi(t)\rangle .
$$

With $\Gamma(t)$ decreasing, accordingly the state vector $|\psi(t)\rangle$ is expected to evolve from the trivial initial ground state of transverse-field term (6) to the nontrivial ground state of (7), which is the solution of the OSTP.

Then an important issue arises, that is, how slowly we should decrease $\Gamma(t)$ to keep the state vector arbitrarily close to the instantaneous ground state of $H_{\text {OSTP }}$. As mentioned in Section 2.3, we will not attempt an actual Schrödinger annealing evolution of the quantum Hamiltonian due to the large Hilbert space. On the contrary, we address the quantum problem by GFMC-QA [25], where annealing will take place in the fictitious time represented by the number of Monte Carlo steps. However, in order to figure out OSTP by GFMCQA, Suzuki-Trotter transformation should be performed in advance, which requires calculation of the matrix elements of an exponential operator between arbitrary configurations $|\psi\rangle$ and $\left\langle\psi^{\prime}\right|$ of the system, a complicated issue for $H_{\mathrm{Kin}}$. Moreover, since the energy gap between the ground state and the first excited state is large at the beginning and decreases with the annealing time, hyperbolic interpolation makes the annealing process more efficient and smoother than the linear one does [18]; we make the strength of the transverse field as following hyperbolic interpolation:

$$
\Gamma(t)=\frac{(1-t / \eta) \zeta}{t / \eta+\xi},
$$

where $\eta$ is the total annealing time and $\zeta, \xi$ are two control operators. This form is trivially Trotter-discretized [18, 19], since the spin-flip term acts independently on single spins at each time slot.

(2) Suzuki-Trotter Transformation. In GFMC-QA, a quantum Hamiltonian is approximated by a classical one with the aid of a Suzuki-Trotter transformation. This is possible because of an analogy with a standard TISG model in a transverse field [13]. The transformation maps the quantum Hamiltonian to an effective classical Hamiltonian $H$ similar to the one mentioned in [24], and then (9) can be rewritten as

$$
\begin{aligned}
H= & \frac{1}{P} \sum_{\rho=1}^{P} H_{\mathrm{TISG}}\left(\left\{\Upsilon_{i, \rho}\right\}\right) \\
& -J_{\Gamma}\left(\sum_{\rho=1}^{P-1} \sum_{i} \Upsilon_{i, \rho} \Upsilon_{i, \rho+1}+\sum_{i} \Upsilon_{i, 1} \Upsilon_{i, P}\right),
\end{aligned}
$$

where $H$ can be viewed as consisting of $P$ replicas $\left\{\Upsilon_{i, \rho}, \rho=\right.$ $1, \ldots, P\}$ of the classical potential energy of a given configuration $H_{\text {pot }}\left(\left\{\Upsilon_{i}\right\}\right)$, with an interaction of a combined kinetic energy between them, and $\Upsilon_{i, \rho}$ denotes the $i$ th spin of the $\rho$ th 


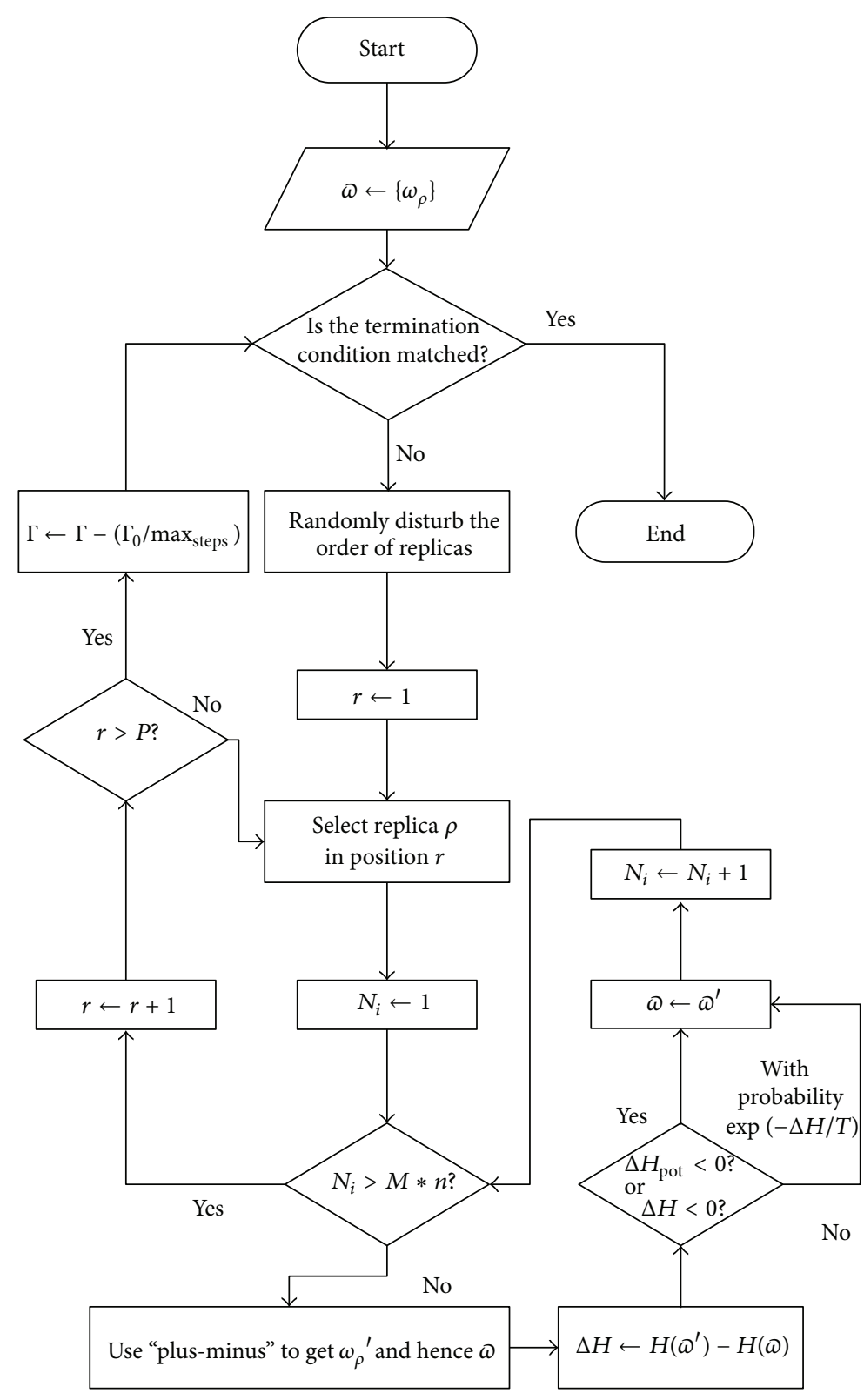

FIGURE 3: Flow chart of QA_OSTP(G, $P, T_{0}, \Gamma_{0}$, Max $\left._{\text {steps }}\right)$.

replica. The term $J_{\Gamma}$ is the coupling among the replicas, which can be written as

$$
J_{\Gamma}=-\frac{T}{2} \ln \tanh \left(\frac{\Gamma(t)}{P T}\right)>0,
$$

where $T$ is the temperature, at which each replica is simulated.

3.2. Proposed QA_OSTP. In this part, we will describe the components of our algorithm in detail. Notations that are used in QA_OSTP are shown in Table 2. Flow chart of QA_ OSTP is given in Figure 3. The first diamond (outermost loop) is controlled by $T$. Here a linear annealing schedule consisting of the initial temperature $T_{0}$ and $\operatorname{Max}_{\text {steps }}$ is selected. Each Monte Carlo step for QA_OSTP consists of a loop starting from the second diamond where $M$ is a tunable multiplier; $M * N$ moves are conducted at each step after which the control parameter is decreased. QA_OSTP keeps making the next Monte Carlo Step each time until the termination condition is satisfied. The replicas are always connected to each other in numerical order in the same way throughout the search for the purpose of spin products. The random disturbing means only changing the order in which replicas are selected for search. We find this as an efficient scheme that can promote diversity in the population of configurations.

3.3. Theoretical Analysis. The convergence conditions for the implementation of QA_OSTP with the quantum Monte Carlo evolution are investigated in this part. 
TABLE 2: Notations used in QA_OSTP.

\begin{tabular}{lc}
\hline$G$ & $\begin{array}{c}\text { Social graph of online social networks with } \\
\text { multiple QoT parameters }\end{array}$ \\
$P$ & The number of replicas \\
$T$ & Temperature parameter \\
$T_{0}$ & Optimal initial temperature \\
$\Gamma_{0}$ & An initial value of the transverse field \\
$M$ & Fixed number of nearest neighbors of participant \\
$N$ & Number of nodes in online social networks \\
$\tau_{\text {total }}$ & Total Monte Carlo time \\
Max $_{\text {steps }}$ & Maximum number of Monte Carlo steps \\
$N_{i}$ & Number of iterations \\
$\omega$ & A social trust path configuration \\
$\omega$ & A series of $\omega$, also known as $\left\{\omega_{\rho}\right\}$ \\
$\omega_{\rho}^{\prime}$ & Neighbor of configuration $\omega_{\rho}$ \\
\hline
\end{tabular}

In our method, the $d$-dimensional TISG is mapped to a $(d+1)$-dimensional classical Ising system. By using the Suzuki-Trotter transformation, at temperature $T$, when the length of the extra dimension $L \rightarrow \infty$, the system partition function can be defined as

$$
\begin{array}{r}
Z_{T}=\sum_{\left\{\omega_{i}^{(k)}\right\}} \exp \left(\frac{1}{\kappa T L} \sum_{k=1}^{L} \sum_{\langle i j\rangle} J_{i j} \delta_{i}^{(k)} \delta_{j}^{(k)}\right. \\
\left.+J_{\Gamma} \sum_{k=1}^{L} \sum_{i=0}^{N} \delta_{i}^{(k)} \delta_{i}^{(k+1)}\right),
\end{array}
$$

where $\kappa$ is Boltzmann's constant, $\delta_{i}^{(k)}$ represents a classical Ising spin at site $i$ on the $k$ th Trotter slice, and $\omega$ is the space of discrete configuration.

A Monte Carlo step is characterized by the transition probability from configuration $\omega_{\rho}$ to configuration $\omega_{\rho}^{\prime}$ at time step $\tau$ :

$$
\mathfrak{I}\left(\omega_{\rho}^{\prime}, \omega_{\rho} ; \tau\right)=P\left(\omega_{\rho}^{\prime}, \omega_{\rho}\right) \Lambda\left(\omega_{\rho}^{\prime}, \omega_{\rho} ; \tau\right)
$$

where $\omega_{\rho}$ is the present configuration, $\omega_{\rho}^{\prime}$ is the next candidate configuration which is generated with defined probability, both of which belong to the space of discrete configuration $\omega$, and $P\left(\omega_{\rho}^{\prime}, \omega_{\rho}\right)$ is the generation probability with which $\omega_{\rho}^{\prime}$ can be generated from $\omega_{\rho}$. The acceptance probability $\Lambda\left(\omega_{\rho}^{\prime}\right.$, $\left.\omega_{\rho} ; \tau\right)$ of QA_OSTP can be written as

$$
\begin{gathered}
\Lambda\left(\omega_{\rho}^{\prime}, \omega_{\rho} ; \tau\right)=\Phi\left(\frac{\Theta\left(\omega_{\rho}^{\prime} ; \tau\right)}{\Theta\left(\omega_{\rho} ; \tau\right)}\right), \\
\Theta\left(\omega_{\rho} ; \tau\right)=\frac{1}{Z_{T}} \exp \left(-\frac{C_{0}\left(\omega_{\rho}\right)}{T_{0}}-\frac{C_{1}\left(\omega_{\rho}\right)}{T_{1}(\tau)}\right),
\end{gathered}
$$

where $C_{0}\left(\omega_{\rho}\right)$ means the cost function whose global minimum is the optimal social trust path of OSTP problem in OSNs, $C_{1}\left(\omega_{\rho}\right)$ represents the kinetic energy, $\Theta\left(\omega_{\rho} ; \tau\right)$ is the stationary distribution of the homogeneous Markov chain defined by matrix $\Im$ at a given $T_{1}(\tau)$, and $\Phi(\cdot)$ is the monotone increasing acceptance function satisfying $0 \leq \Phi(\cdot) \leq 1$.

To derive the convergence conditions for the implementation of QA_OSTP with the quantum Monte Carlo evolution, we should prove that inhomogeneous Markov chain associated with QA_OSTP is strongly ergodic under appropriate conditions [28]. Our main results are summarized in the following theorems.

Theorem 4. For a causal system, the transition matrix $\mathfrak{I}$ has the following lower bound:

$$
\Im\left(\omega_{\rho}^{\prime}, \omega_{\rho} ; \tau\right) \geq p \Phi(1) \exp \left(-\frac{L_{0}}{T_{0}}-\frac{L_{1}}{T_{1}(\tau)}\right)
$$

where $p$ is the minimum nonvanishing value of $P\left(\omega_{\rho}^{\prime}, \omega_{\rho}\right)$, and $L_{0}$ and $L_{1}$ are the maximum changes in a single step in $C_{0}\left(\omega_{\rho}\right)$ and $C_{1}\left(\omega_{\rho}\right)$, respectively.

Proof. Directly following the definition of the transition probability and the property of the acceptance function in (15), (16), and (17), for both positive $L_{0}$ and $L_{1}$, if $\Theta\left(\omega_{\rho}^{\prime} ; \tau\right) / \Theta$ $\left(\omega_{\rho} ; \tau\right)<1$, we get

$$
\begin{aligned}
\Im\left(\omega_{\rho}^{\prime}, \omega_{\rho} ; \tau\right) & \geq p \Phi\left(\frac{\Theta\left(\omega_{\rho} ; \tau\right)}{\Theta\left(\omega_{\rho}^{\prime} ; \tau\right)}\right) \frac{\Theta\left(\omega_{\rho}^{\prime} ; \tau\right)}{\Theta\left(\omega_{\rho} ; \tau\right)} \\
& \geq p \Phi(1) \exp \left(-\frac{L_{0}}{T_{0}}-\frac{L_{1}}{T_{1}(\tau)}\right)
\end{aligned}
$$

else, we have

$$
\begin{aligned}
\mathfrak{\Im}\left(\omega_{\rho}^{\prime}, \omega_{\rho} ; \tau\right) & \geq p \Phi(1) \\
& \geq p \Phi(1) \exp \left(-\frac{L_{0}}{T_{0}}-\frac{L_{1}}{T_{1}(\tau)}\right) .
\end{aligned}
$$

Theorem 4 is thereby proved.

Theorem 5. The inhomogeneous Markov chain generated by (16) and (17) is strongly ergodic and converges to the equilibrium state, which is corresponding to the term $\exp \left(-C_{0}\left(\omega_{\rho}\right) /\right.$ $\left.T_{0}\right)$ in (17).

Proof. In order to prove strong ergodicity, we refer to the conditions for strong ergodicity [28]. If there exists the transition matrix $\mathbb{N}$ on $\omega$ such that $\mathbb{N}\left(\omega_{\rho}^{\prime \prime}, \omega_{\rho}\right)=\mathbb{N}\left(\omega_{\rho}^{\prime \prime}, \omega_{\rho}^{\prime}\right)$ for any $\omega_{\rho}$, $\omega_{\rho}^{\prime}, \omega_{\rho}^{\prime \prime} \in \Phi$, then for a causal system, we have

$$
\lim _{\tau \rightarrow \infty}\left\|\mathfrak{J}^{\tau, \omega}-\mathbb{N}\right\|=0
$$

Since the Markov chain is proved to be weakly ergodic in [28], we consider for any $s>0, p(\tau, s)=\mathfrak{\Im}^{\tau, \oplus} p_{0}$, where $p_{0}$ belongs to the set of probability distributions on $\omega_{\rho} \in \omega$. For 


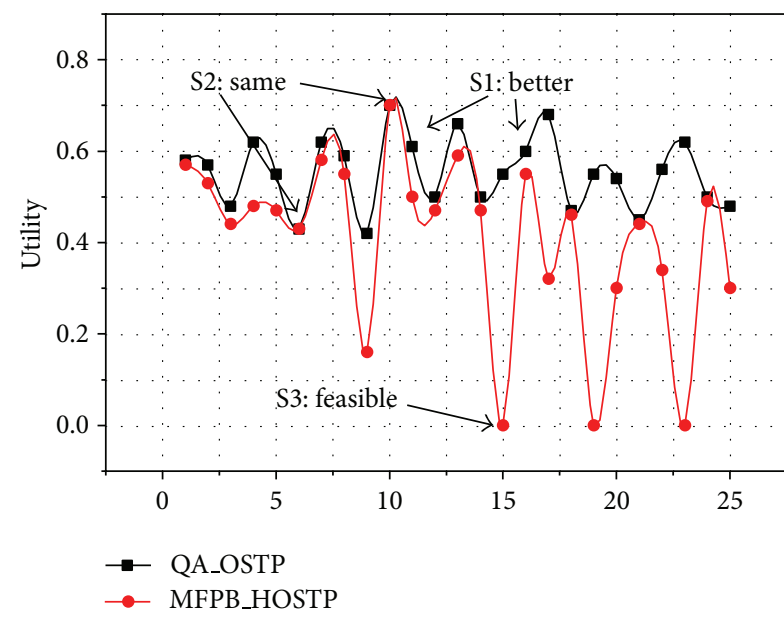

(a) Network ID with WID = 1

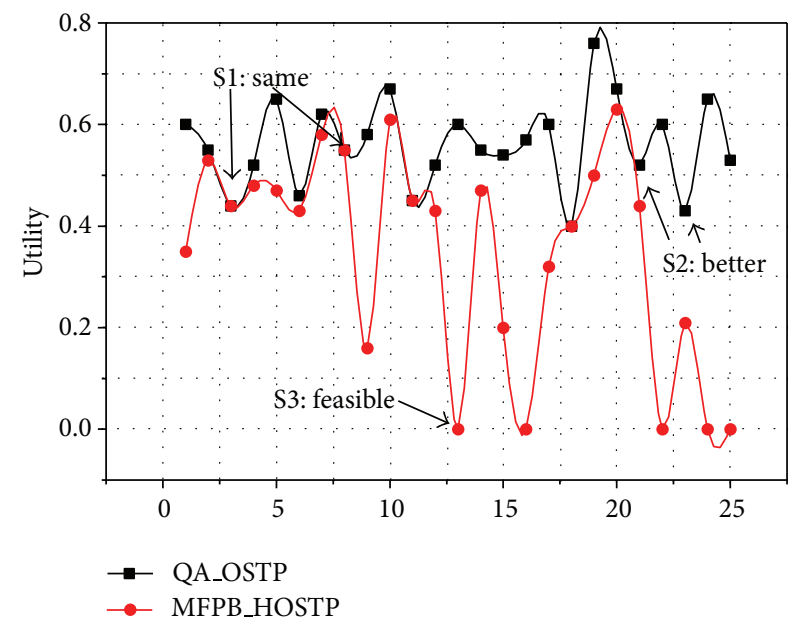

(c) Network ID with WID $=3$

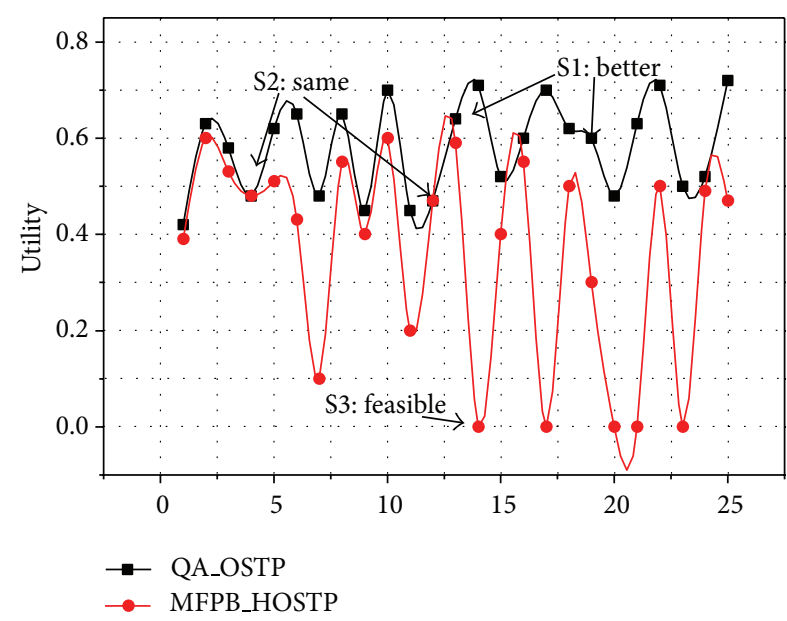

(b) Network ID with WID = 2

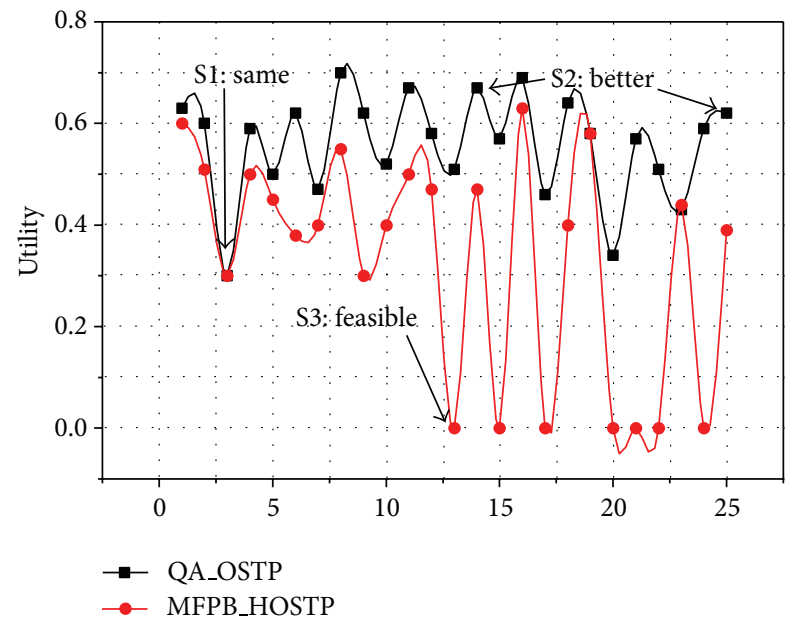

(d) Network ID with WID $=4$

FIGURE 4: Comparison of path utilities of networks.

a fixed $\omega \in \emptyset$, the probability distribution can be written as $p\left(\omega_{\rho}^{\prime \prime}\right)=\mathbb{N}\left(\omega_{\rho}^{\prime \prime}, \omega\right)$. Then we have

$$
\begin{aligned}
& \|p(\tau, \omega)-p\| \\
& \quad=\sum_{\omega_{\rho}^{\prime \prime} \in \Phi}\left|\sum_{\omega_{\rho} \in \Phi} \mathfrak{J}^{\tau, \Phi}\left(z, \omega_{\rho}\right) p_{0}\left(\omega_{\rho}\right)-\mathbb{N}\left(\omega_{\rho}^{\prime \prime}, \omega\right)\right| \\
& \leq \sum_{\omega_{\rho}^{\prime \prime} \in \Phi}\left|\sum_{\omega_{\rho} \in \Phi}\left[\mathfrak{J}^{\tau, \Phi}\left(\omega_{\rho}^{\prime \prime}, \omega_{\rho}\right)-\mathbb{N}\left(\omega_{\rho}^{\prime \prime}, \omega\right)\right] p_{0}\left(\omega_{\rho}\right)\right| \\
& \leq \sum_{\omega_{\rho} \in \Phi}\left\|\mathfrak{J}^{\tau, \omega}-\mathbb{N}\right\|=|\Phi|\left\|\mathfrak{J}^{\tau, \omega}-\mathbb{N}\right\| ;
\end{aligned}
$$

then when $\tau \rightarrow \infty$, based on (21), we get

$$
\sup \left\{\left\|p(\tau, s)-p_{\omega}\right\|\right\}=0 .
$$

Therefore, the inhomogeneous Markov chain generated by (16) and (17) is strongly ergodic and thereby is proved.

\section{Experimental Results}

4.1. Experiment Settings. In our experiments, if no otherwise specified, all the related parameters are set following reference [5]. In order to evaluate our proposed algorithm, we compare QA_OSTP with MFPB_HOSTP in terms of two key factors, that is, execution time and the utility of the selected social trust path.

For QA_OSTP, we implement a similar GFMC that was used in [25] at a fixed low temperature $T$ (we used $T=10 / 3$ ). The quantum model is mapped onto a classical model with an extra imaginary-time dimension, consisting of $P$ ferromagnetically coupled replicas of the original spin problem, at temperature $P T$ [25] (we used $P=30$ ). Since QA requires initial configurations equilibrated at temperature $P T$, an obvious choice is to take $P T=100$ [25]. Finally, the transverse field $\Gamma$ is annealed hyperbolically in a MC time $\tau$ from an initial value $\Gamma_{0}=300$ to a final value of zero. In QA, we used exclusively "plus-minus" tactics, with a static neighborhood pruning [29], which restricts the attempted neighborhood realization by allowing only a fixed number $M$ (we used $M=20$ ) of 


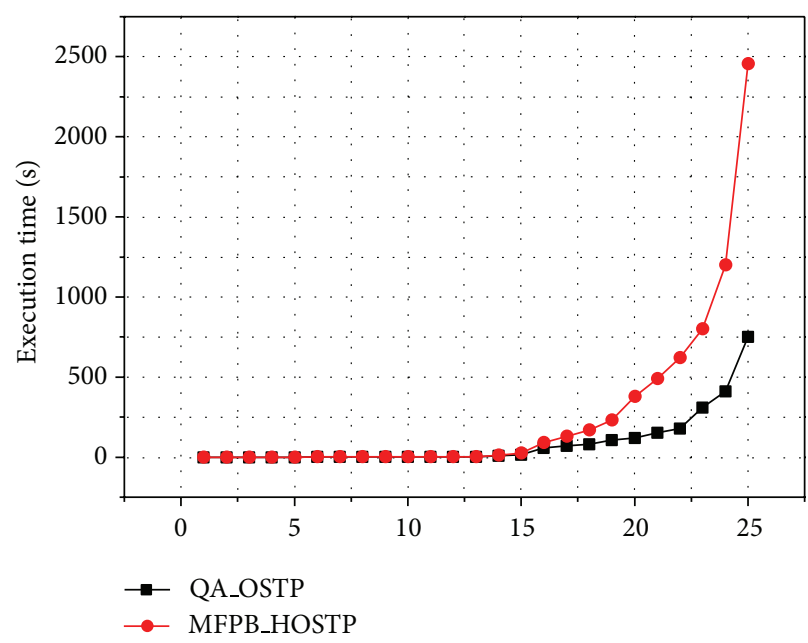

(a) Network ID with WID $=1$

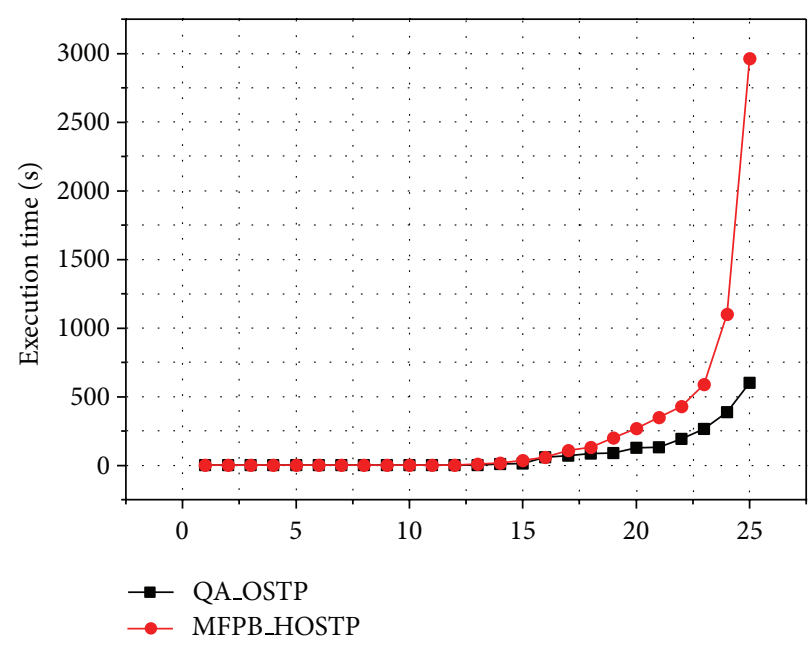

(c) Network ID with WID $=3$

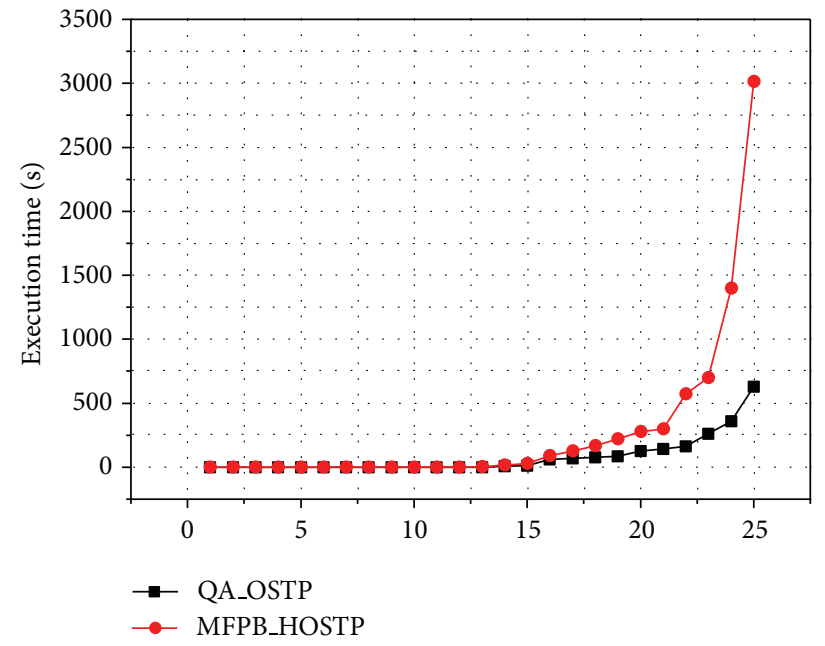

(b) Network ID with WID $=2$



(d) Network ID with WID $=4$

FIGURE 5: Comparison of execution time of algorithms.

nearest neighbors of participant $j$ to be candidates for $j^{\prime}$. Our MC step consisted of $M * N$ attempted operations of "plusminus" tactics (for QA, in each of the $P$ replicas). In QA, we averaged the best social trust path utility found over up to 100 independent searches.

In our experiments, the three QoT parameters are randomly generated. The end-to-end QoT constraints specified by a source participant are set as $V^{T} \geq 0.05, V^{r} \geq 0.001$, and $V^{\rho} \geq 0.3$, respectively. We first randomly select 80 pairs of source and target participants from the Enron email dataset with 87,474 nodes and 30,0511 links. Moreover, following the small world characteristic we set the maximal length of a social trust path 6 hops. Then we number the different network scales from 1 to 25 , with number of nodes varying from 50 to 400 and number of links varying from 63 to 2356 , respectively.

Both algorithms were implemented in Matlab 7.0 and run on a $\mathrm{PC}$ with a $3 \mathrm{GHz}$ Intel processor and $3 \mathrm{~GB}$ of RAM with Windows 7.
4.2. Performance Analysis. Figure 4 plots the utilities of the selected social trust paths with different network scales and different weights of QoT constraints. The weights of QoT parameters are set as $w_{T}=0.25,0.25,0.5,0.3, w_{r}=0.25,0.5$, $0.25,0.3, w_{\rho}=0.5,0.25,0.25,0.4$, and each group corresponds to Weight ID 1,2,3,4, respectively. From the figure, we can observe that our QA_OSTP can always find utilities that are not worse than those of MFPB_HOSTP (e.g., case S1 and S2). This is because in QA_OSTP, quantum fluctuation is adopted to avoid the local minima and quantum mechanics works with wave functions that can sample different regions of phase space equally well. While for MFPB HOSTP, although subnetworks are extracted through exhaustive searching before the algorithm execution and Backward_Search scheme is used to estimate whether there exists a feasible solution in a subnetwork, due to the intrinsic characteristic of heuristic algorithms, it does not guarantee that the best social trust path will be found. Moreover with the network scale growing larger, when the social trust path with 
the maximal utility is not a feasible solution, the heuristic search can hardly find a near optimal solution and usually returns an infeasible one even when a feasible solution exists (e.g., case S3). Thus, in any case, QA_OSTP shows better performance. In particular, we find that the mean value of utilities of QA_OSTP is $42.85 \%$ more than that of MFPB_HOSTP in Figure 4(a), 58.50\% more in Figure 4(b), 55.84\% more in Figure 4(c), and 63.12\% more in Figure 4(d).

Figure 5 shows the average execution time of algorithms with different weights of QoT parameters and different network scales. Note that, for MFPB_HOSTP, execution time should include the exhaustive searching time for extracting subnetworks, which is not taken into consideration in reference [5]. From Figure 5, we can see when the network scale is not large, both MFPB_HOSTP and QA_OSTP perform well and the difference is trivial because the searching space is relatively small. However, with the network scale expanding, we can observe that QA_OSTP can outperform MFPB_HOSTP in execution time. This is an interesting result since from conventional point, annealing process may be a little time-consuming. The reason we think is that the quantum Hamiltonian in QA_OSTP is approximated with the aid of a SuzukiTrotter transformation in GFMC-QA and quantum tunneling can avoid some unnecessary searching in MFPB_HOSTP, which can accelerate the annealing process. From Figure 5, we can see that the average execution time of our proposed algorithm is only $33.47 \%$ of that of MFPB_HOSTP in Figure 5(a), 28.36\% in Figure 5(b), 31.99\% in Figure 5(c), and $26.78 \%$ in Figure 5(d).

Based on the above experiments conducted with different scales and parameters, we can observe that QA_OSTP is a promising algorithm and it performs better than its heuristic opponent in both the quality of the selected social trust path and the execution time.

\section{Conclusion}

A novel quantum annealing based OSTP algorithm, that is, QA_OSTP for selection of the most trustworthy path to service provider in online social networks, was proposed. To the best our knowledge, this is the first application of quantum annealing to the challenging NP-Complete OSTP problem in online social networks. Since quantum mechanics work with wave functions that can sample different regions of phase space equally well and quantum systems can tunnel through classically impenetrable potential barriers between energy valleys, a process that might prove more effective than waiting for them to be overcome thermally as in SA, QA_OSTP is able to outperform its heuristic opponents and even find configuration of comparable quality to the best algorithm. Thus, QA_OSTP is proven to be a very promising tool for solving the OSTP in online social networks.

As for the future work, understanding how quantum mechanics can quantitatively improve the quality of solution of OSTP is still an important open issue. Moreover, since GFMC simulations using quantum-classical mapping with the aid of a Suzuki-Trotter transformation only simulate the equilibrium behavior at finite temperature, we plan to devise another effective and alternative scheme to solve the infinite time Schrödinger equation with stochastic processes.

\section{Acknowledgments}

This work is supported partially by National Natural Science Foundation of China (NSFC) under Grant nos. 61002016 and 61101111, Zhejiang Provincial Natural Science Foundation of China under Grant no. LY13F010016, and Qianjiang Talent Project of Zhejiang Province under Grant no. QJD1302014.

\section{References}

[1] R.-C. Chen, "Grouping optimization based on social relationships," Mathematical Problems in Engineering, vol. 2010, Article ID 170563, 19 pages, 2012.

[2] R. Chen, E. K. Lua, and Z. Cai, "Bring order to online social networks," in Proceedings of 30th Annual Joint Conference of the IEEE Computer and Communications Societies (INFOCOM '11), pp. 541-545, Shanghai, China, April 2011.

[3] Social Networks \& Blogs Now 4th Most Popular Online Activity, Ahead of Personal Email, Nielsen Reports, http://www .nielsen.com/us/en/press-room/2009/social_networks_..html.

[4] J. Kunegis, A. Lommatzsch, and C. Bauckhage, "The slashdot zoo: mining a social network with negative edges," in Proceedings of the 18th International Conference on World Wide Web (WWW '09), pp. 741-750, ACM, Madrid, Spain, April 2009.

[5] G. Liu, Y. Wang, M. A. Orgun, and E. -P. Lim, "Finding the optimal social trust path for the selection of trustworthy service providers in complex social networks," IEEE Transactions on Services Computing, vol. 6, no. 2, pp. 152-167, 2011.

[6] S. Baase, Computer Algorithms: Introduction to Design \& Analysis, 3/E, Pearson Education India, 2000.

[7] T. Korkmaz and M. Krunz, "Multi-constrained optimal path selection," in Proceedings of the 20th Annual Joint Conference of the IEEE Computer and Communications Societies (INFOCOM '01), vol. 2, pp. 834-843, Anchorage, Alaska, USA, April 2001.

[8] T. Yu, Y. Zhang, and K.-J. Lin, "Efficient algorithms for web services selection with end-to-end QoS constraints," ACM Transactions on the Web, vol. 1, no. 1, article 6, 2007.

[9] E. W. Dijkstra, "A note on two problems in connexion with graphs," Numerische Mathematik, vol. 1, pp. 269-271, 1959.

[10] P. J. van Laarhoven and E. H. Aarts, Simulated Annealing: Theory and Applications, Springer, 1987.

[11] D. T. Pham and D. Karaboga, Intelligent Optimisation Techniques: Genetic Algorithms, Tabu Search, Simulated Annealing and Neural Networks, Springer, New York, NY, USA, 2000.

[12] S. Kirkpatrick, C. D. Gelatt, Jr., and M. P. Vecchi, "Optimization by simulated annealing," Science, vol. 220, no. 4598, pp. 671-680, 1983.

[13] A. Das and B. K. Chakrabarti, "Colloquium: quantum annealing and analog quantum computation," Reviews of Modern Physics, vol. 80, no. 3, pp. 1061-1081, 2008.

[14] J. Brooke, D. Bitko, T. F. Rosenbaum, and G. Aeppli, "Quantum annealing of a disordered magnet," Science, vol. 284, no. 5415, pp. 779-781, 1999.

[15] H. M. Rønnow, R. Parthasarathy, J. Jensen, G. Aeppli, T. F. Rosenbaum, and D. F. McMorrow, "Quantum phase transition of a magnet in a spin bath," Science, vol. 308, no. 5720, pp. 389$392,2005$.

[16] Y. Matsuda, H. Nishimori, and H. G. Katzgraber, Quantum Annealing for Problems With Ground-State Degeneracy, IOP Publishing, 2003. 
[17] T. Kadowaki and H. Nishimori, "Quantum annealing in the transverse Ising model," Physical Review E, vol. 58, no. 5, pp. 5355-5363, 1998.

[18] S. Suzuki, J.-I. Inoue, and B. K. Chakrabarti, "Quantum annealing," in Quantum Ising Phases and Transitions in Transverse Ising Models, pp. 225-289, 2nd edition, 2013.

[19] S. Boixo, "Experimental signatures of quantum annealing," Bulletin of the American Physical Society, article 58, 2013.

[20] W. W. Cohen, Enron email dataset. Internet, 2009, http://www .cs.cmu.edu/ enron/.

[21] S. Yoo, Y. Yang, F. Lin, and C. Moon, "Mining social networks for personalized email prioritization," in Proceedings of the 15th ACM SIGKDD International Conference on Knowledge Discovery and Data Mining (KDD '09), pp. 967-975, July 2009.

[22] A. McCallum, X. Wang, and A. Corrada-Emmanuel, "Topic and role discovery in social networks with experiments on enron and academic email," Journal of Artificial Intelligence Research, vol. 30, pp. 249-272, 2007.

[23] J. Goldstein, A. Kwasinski, P. Kingsbury, R. E. Sabin, and A. Mcdowell, "Annotating subsets of the enron email corpus," CiteSeer, http://citeseerx.ist.psu.edu/viewdoc/summary?doi= 10.1.1.89.4060.

[24] D. A. Battaglia, G. E. Santoro, and E. Tosatti, "Optimization by quantum annealing: lessons from hard satisfiability problems," Physical Review E, vol. 71, no. 6, Article ID 066707, 10 pages, 2005.

[25] D. P. Landau and K. Binder, A Guide to Monte Carlo Simulations in Statistical Physics, Cambridge University Press, Cambridge, UK, 3rd edition, 2009.

[26] B. K. Chakrabarti, A. Dutta, and P. Sen, "Quantum Ising phases and transitions in transverse Ising models," in Quantum Ising Phases and Transitions in Transverse Ising Models, vol. 41 of Lecture Notes in Physics Monographs, Springer, Berlin, Germany, 1996.

[27] L. Liu and G. Feng, "Simulated annealing based multi-constrained QoS routing in mobile ad hoc networks," Wireless Personal Communications, vol. 41, no. 3, pp. 393-405, 2007.

[28] B. K. Chakrabarti and A. Das, Quantum Annealing and Related Optimization Methods, Springer, 2005.

[29] E. Aarts and J. K. Lenstra, Local Search in Combinatorial Optimization, Princeton University Press, Princeton, NJ, USA, 2003. 


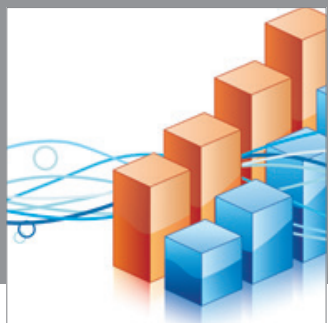

Advances in

Operations Research

mansans

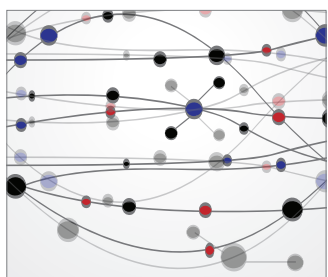

The Scientific World Journal
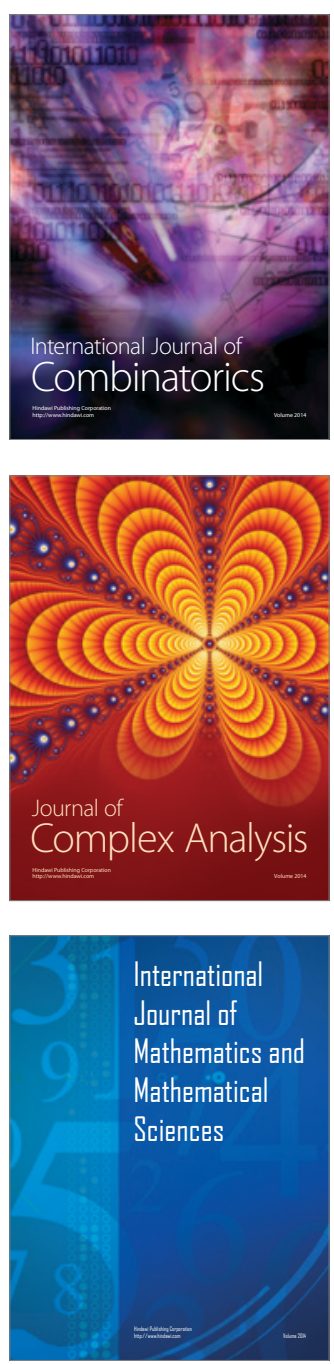
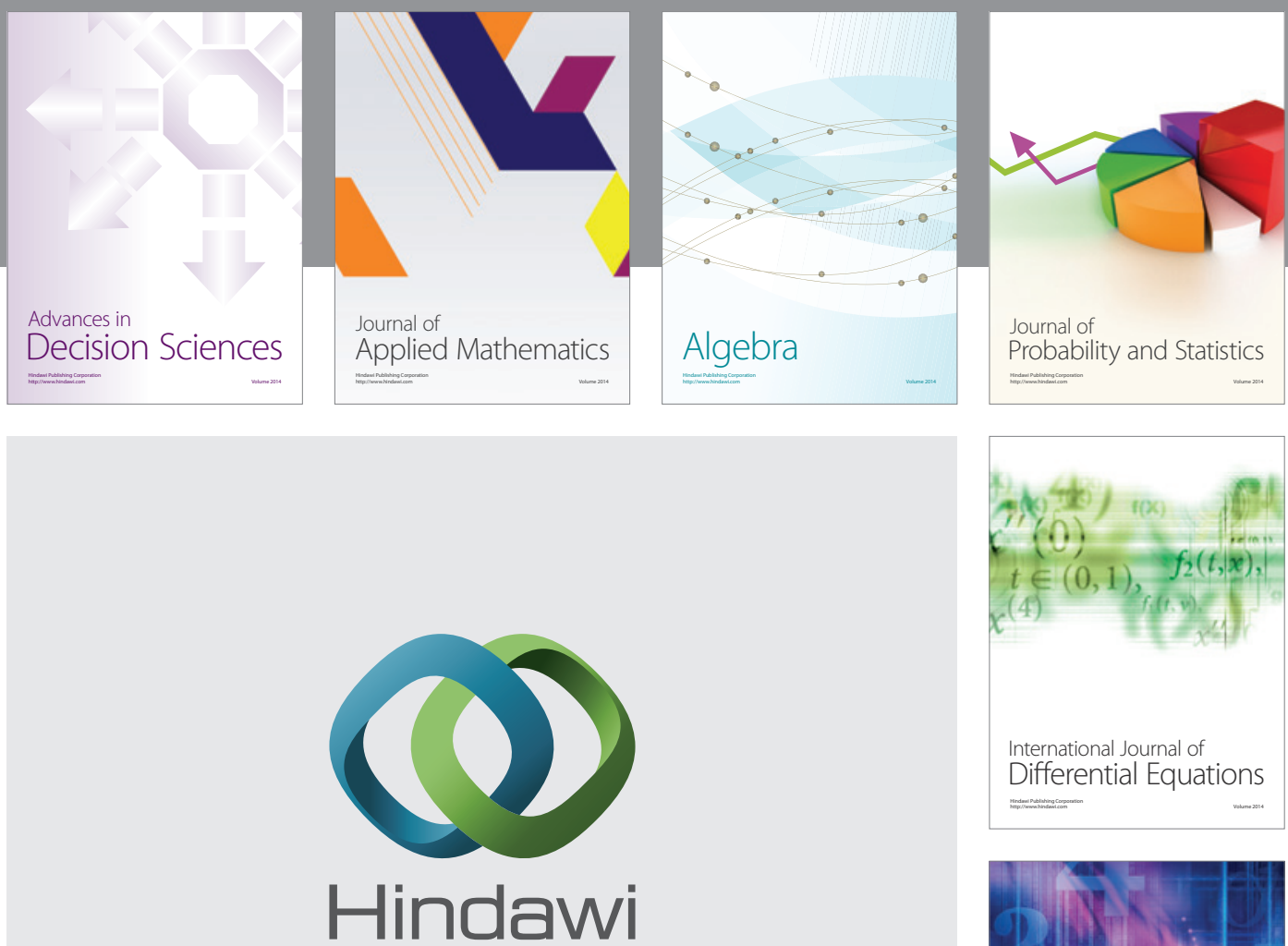

Submit your manuscripts at http://www.hindawi.com
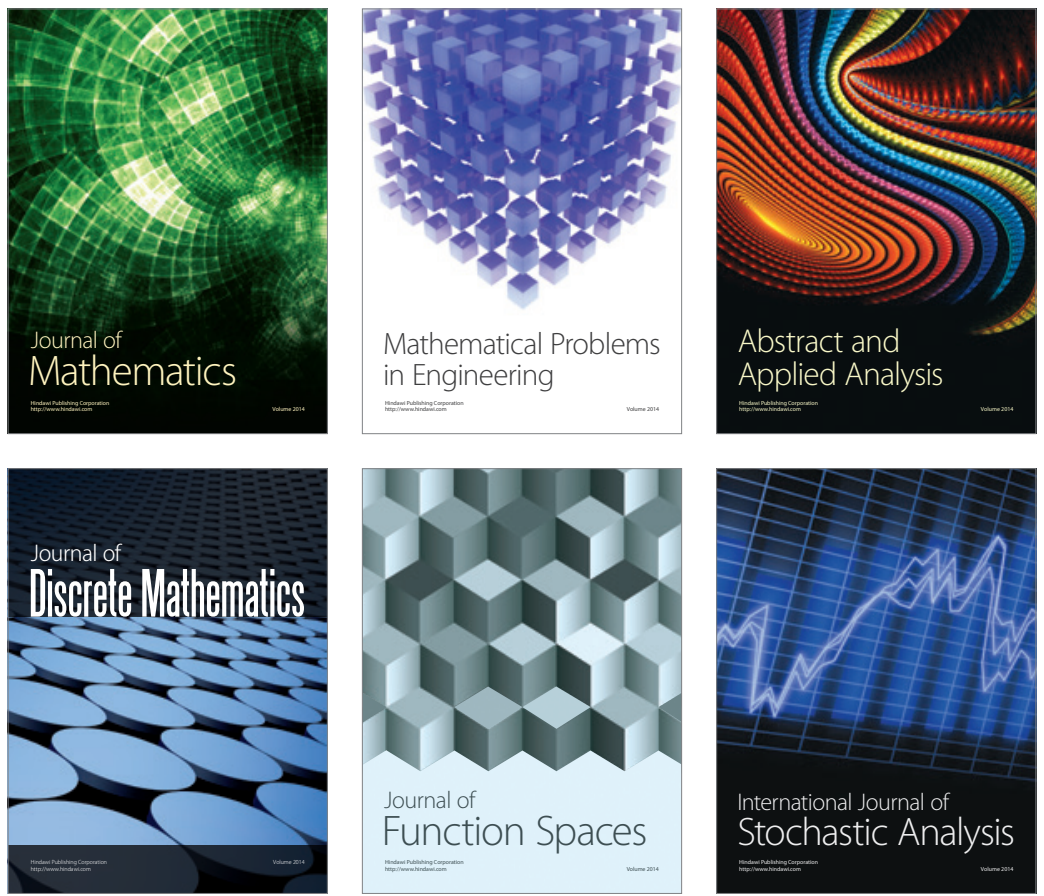

Journal of

Function Spaces

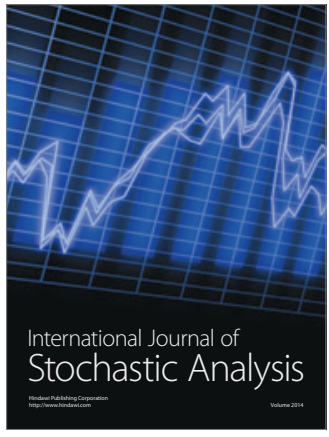

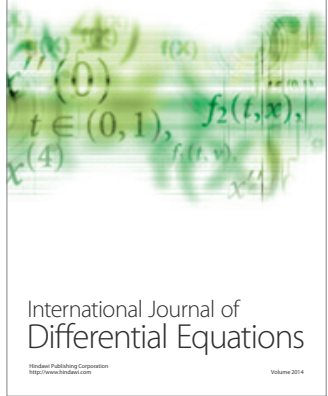
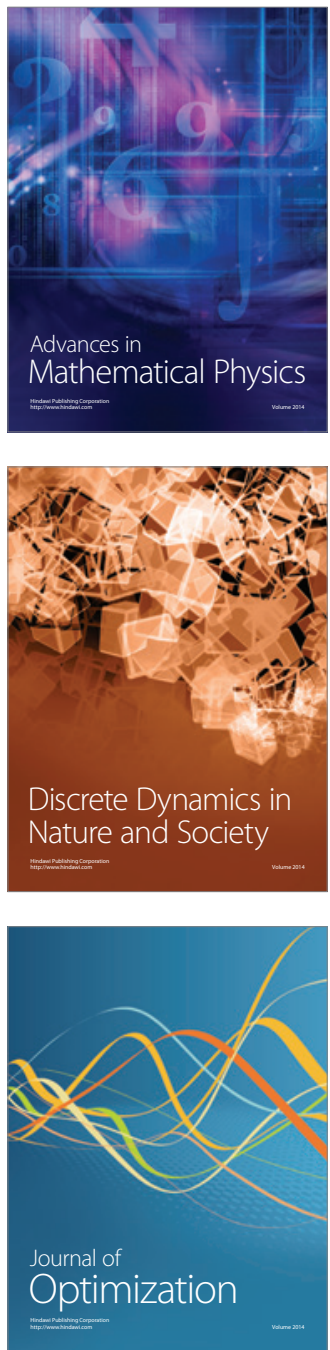